$E_{\mathrm{F}}$ and $k_{\mathrm{F}}$ feature in the geometry. An early indication of such scales was seen in $(24,36)$ in a variant of the story that geometry is not universal in string theory: The geometry depends on the probe used, and different probes experience different geometric backgrounds. The absence of these scales in the general relativistic description of the AdS black hole could thus be an artifact of the Riemannian metric description of space-time.

Regardless of these questions, AdS/CFT has shown itself to be a powerful tool to describe finite-density Fermi systems. The description of the emergent Fermi liquid presented here argues that AdS/CFT is uniquely suited as a computational device for field theory problems suffering from fermion sign problems. AdS/CFT represents a rich mathematical environment and a new approach to qualitatively and quantitatively investigate important questions in quantum manybody theory at finite fermion density.

\section{References and Notes}

1. M. Troyer, U. J. Wiese, Phys. Rev. Lett. 94, 170201 (2005).

2. S. Sachdev, Quantum Phase Transitions (Cambridge Univ. Press, Cambridge, 1999).

3. J. Zaanen, Science 319, 1295 (2008).

4. D. van der Marel et al., Nature 425, 271 (2003).

5. ]. Zaanen, Nature 430, 512 (2004).

6. T. Senthil, Phys. Rev. B 78, 035103 (2008)

7. F. Krüger, ]. Zaanen, Phys. Rev. B 78, 035104 (2008).
8. D. T. Son, A. O. Starinets, Annu. Rev. Nucl. Part. Sci. 57, 95 (2007).

9. S. S. Gubser, A. Karch, http://arxiv.org/abs/0901.0935 (2009).

10. C. P. Herzog, P. Kovtun, S. Sachdev, D. T. Son, Phys. Rev. D 75, 085020 (2007).

11. S. A. Hartnoll, P. K. Kovtun, M. Muller, S. Sachdev, Phys. Rev. B 76, 144502 (2007)

12. S. S. Gubser, Phys. Rev. D 78, 065034 (2008).

13. S. A. Hartnoll, C. P. Herzog, G. T. Horowitz, Phys. Rev. Lett. 101, 031601 (2008).

14. S. A. Hartnoll, C. P. Herzog, G. T. Horowitz, J. High Energy Phys. 0812, 015 (2008)

15. D. T. Son, Phys. Rev. D 78, 046003 (2008).

16. K. Balasubramanian, ]. McGreevy, Phys. Rev. Lett. 101, 061601 (2008).

17. A. Adams, K. Balasubramanian, ]. McGreevy, ]. High Energy Phys. 0811, 059 (2008).

18. S. A. Hartnoll, Science 322, 1639 (2008)

19. S. A. Hartnoll, http://arxiv.org/abs/0903.3246 (2009)

20. M. Kulaxizi, A. Parnachev, Nucl. Phys. B 815, 125 (2009).

21. A. Karch, D. T. Son, A. O. Starinets, Phys. Rev. Lett. 102 , 051602 (2009)

22. M. Kulaxizi, A. Parnachev, Phys. Rev. D 78, 086004 (2008).

23. L. Brits, M. Rozali, http://arxiv.org/abs/0810.5321 (2008).

24. H. H. Shieh, G. van Anders, J. High Energy Phys. 0903, 019 (2009)

25. See supporting material on Science Online.

26. ARPES Fermi-surface measurements assume that electrons are the only relevant charged objects. If this is so, then it measures the electron (i.e., fermion) spectral function. This spectral function is what we compute here, even though in our AdS setup the fermions are almost certainly not the only charged objects.

27. H. Liu, ]. McGreevy, D. Vegh, http://arxiv.org/abs/ 0903.2477 (2009)
28. S. S. Lee, Phys. Rev. D 79, 086006 (2009)

29. E. M. Lifshitz, L. P. Pitaevskii, Statistical Physics, Part 2 (Pergamon, Oxford, 1980).

30. H. J. Schulz, G. Cuniberti, P. Pieri, in Field Theories for Low-Dimensional Condensed Matter Systems, G. Morandi, P. Sodano, A. Tagliacozzo, V. Tognetti, Eds. (Springer, Berlin, 2000), chap. 2.

31. M. Randeria, A. Paramekanti, N. Trivedi, Phys. Rev. B 69, 144509 (2004).

32. Note that the $m=0$ spectral peak discussed in (27) is therefore not the peak we identified with the quasi-particle state. See (25).

33. M. J. Lawler, V. Fernandez, D. G. Barci, E. Fradkin, L. Oxman, Phys. Rev. B 73, 085101 (2006).

34. P. K. Kovtun, A. O. Starinets, Phys. Rev. D 72, 086009 (2005).

35. H. T. Cho, A. S. Cornell, ]. Doukas, W. Naylor, Phys. Rev. D 77, 016004 (2008).

36. M. Rozali, H. H. Shieh, M. Van Raamsdonk, J. Wu, J. High Energy Phys. 0801, 053 (2008).

37. We thank F. Denef, S. Hartnoll, H. Liu, ]. McGreevy, S. Sachdev, D. Sadri, and D. Vegh for discussions. Supported by a VIDI Innovative Research Incentive Grant (K.S.) from the Netherlands Organization for Scientific Research (NWO) and by a Spinoza Award (J.Z.) from NWO and the Dutch Foundation for Fundamental Research on Matter (FOM)

Supporting Online Material

www.sciencemag.org/cgi/content/full/1174962/DC1

SOM Text

Fig. S1

References

14 April 2009; accepted 16 June 2009

Published online 25 June 2009;

$10.1126 /$ science. 1174962

Include this information when citing this paper.

\title{
Radio Imaging of the Very-High-Energy $\gamma$-Ray Emission Region in the Central Engine of a Radio Galaxy
}

\author{
The VERITAS Collaboration, the VLBA $43 \mathrm{GHz}$ M87 Monitoring Team, \\ the H.E.S.S. Collaboration, the MAGIC Collaboration*
}

The accretion of matter onto a massive black hole is believed to feed the relativistic plasma jets found in many active galactic nuclei (AGN). Although some AGN accelerate particles to energies exceeding $10^{12}$ electron volts and are bright sources of very-high-energy (VHE) $\gamma$-ray emission, it is not yet known where the VHE emission originates. Here we report on radio and VHE observations of the radio galaxy Messier 87, revealing a period of extremely strong VHE $\gamma$-ray flares accompanied by a strong increase of the radio flux from its nucleus. These results imply that charged particles are accelerated to very high energies in the immediate vicinity of the black hole.

A ctive galactic nuclei (AGN) are extragalactic objects thought to be powered by massive black holes in their centers. They can show strong emission from the core, which is often dominated by broadband continuum radiation ranging from radio to $\mathrm{x}$-rays and by substantial flux variability on different time scales. More than 20 AGN have been es-

*The full list of authors and affiliations is presented at the end of this paper. tablished as very-high-energy (VHE) $\gamma$-ray emitters with measured energies above $0.1 \mathrm{TeV}$; the jets of most of these sources are believed to be aligned with the line of sight to within a few degrees. The size of the VHE $\gamma$-ray emission region can generally be constrained by the time scale of the observed flux variability $(1,2)$, but its location remains unknown.

We studied the inner structure of the jet of the giant radio galaxy Messier 87 (M87), a known VHE $\gamma$-ray-emitting AGN (2-5) with a $(6.0 \pm$
$0.5) \times 10^{9}$ solar mass black hole (6) (scaled by distance), located $16.7 \mathrm{Mpc}$ (54 million light years) away in the Virgo cluster of galaxies. The angle between its plasma jet and the line of sight is estimated to lie between $15^{\circ}$ and $25^{\circ}$ [see supporting online material (SOM) text]. The substructures of the jet, which are expected to scale with the Schwarzschild radius $R_{\mathrm{S}}$ of the black hole (7), are resolved in the x-ray, optical, and radio wave bands (8) (Fig. 1). High-frequency radio very-long-baseline interferometry (VLBI) observations with resolution under a milli-arc second (milli-arc sec) are starting to probe the collimation region of the jet (9). With its proximity, bright and wellresolved jet, and very massive black hole, M87 provides a unique laboratory in which to study relativistic jet physics in connection with the mechanisms of VHE $\gamma$-ray emission in AGN.

VLBI observations of the M87 inner jet show a well-resolved, edge-brightened structure extending to within 0.5 milli-arc sec $(0.04 \mathrm{pc}$ or $70 R_{\mathrm{s}}$ ) of the core. Closer to the core, the jet has a wide opening angle, suggesting that this is the collimation region (9). Generally, the core can be offset from the actual location of the black hole by an unknown amount (10), in which case it could mark the location of a shock structure or the region where the jet becomes optically thin. However, in the case of M87, a weak structure 
is seen on the opposite side of the core from the main jet, which may be the counter-jet, based on its morphology and length $(11,12)$. Together with the observed pattern in opening angles, this suggests that the black hole of M87 is located within the central resolution element of the VLBI images, at most a few tens of $R_{\mathrm{s}}$ from the radio core (see SOM text). Along the jet, previous monitoring observations show both near-stationary components (12) (parsec scale) and features that

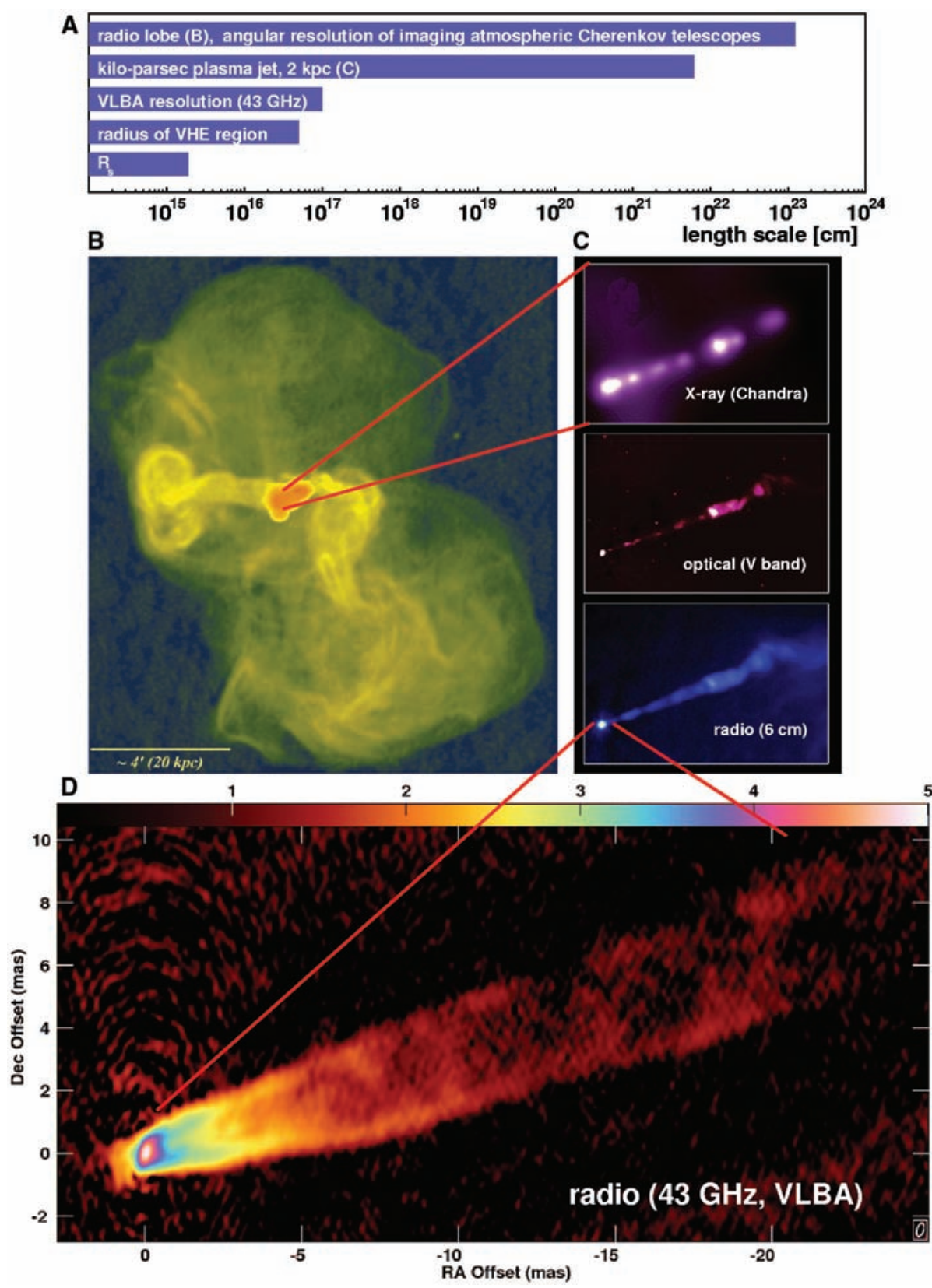

Fig. 1. M87 at different photon frequencies and length scales. (A) Comparison of the different length scales. (B) $90-\mathrm{cm}$ radio emission measured with the VLA. The jet outflows terminate in a halo that has a diameter of $\sim 80 \mathrm{kpc}\left(15^{\prime}\right)$. The radio emission in the central region is saturated in this image. [Credit: F. N. Owen, ]. A. Eilek, and N. E. Kassim (32), NRAO/Associated Universities Incorporated/NSF] (C) Zoomed image of the plasma jet with an extension of $2 \mathrm{kpc}\left(20^{\prime \prime}\right)$, seen in different frequency bands: $\mathrm{x}$-rays (Chandra, top), optical (V band, middle), and radio $(6 \mathrm{~cm}$, bottom). Individual knots in the jet and the nucleus can be seen in all three frequency bands. The innermost knot HST-1 is located at a projected distance of 0.86 arc sec $\left(60 \mathrm{pc}, \approx 10^{5} R_{\mathrm{s}}\right)$ from the nucleus. [Credit: $\mathrm{x}$-ray, NASA/Chandra X-Ray Observatory Science Center/Massachusetts Institute of Technology/H. Marshall et al.; radio, F. Zhou, F. Owen (NRAO), ]. Biretta (Space Telescope Science Institute); optical, NASASSTScI/University of Maryland Baltimore County/E. Perlman et al. (8)] (D) An averaged, and hence smoothed, radio image based on 23 images from the VLBA monitoring project at $43 \mathrm{GHz}$. The color scale gives the logarithm of the flux density in units of 0.01 millijansky per beam. The indication of a counter-jet can be seen, emerging from the core toward the lower left side. mas, milli-arc seconds.

move at apparent superluminal speeds $(13,14)$ (100 pc scale). The presence of superluminal motions and the strong asymmetry of the jet brightness indicate that the jet flow is relativistic. The near-stationary components could be related to shocks or instabilities that can be either stationary (for example, if they are the result of interaction with the external medium) or slowly moving (if they are the result of instabilities in the flow).

A first indication of VHE $\gamma$-ray emission from M87 was reported by the High Energy Gamma-Ray Astronomy (HEGRA) Collaboration in 1998/1999 (3). The emission was confirmed by the High Energy Stereoscopic System (H.E.S.S.) in 2003 to 2006 (2), with $\gamma$-ray flux variability on time scales of days. M87 was detected again with the Very Energetic Radiation Imaging Telescope Array System (VERITAS) in 2007 (4) and, recently, the short-term variability was confirmed with the Major Atmospheric Gamma-Ray Imaging Cherenkov (MAGIC) telescope during a strong VHE $\gamma$-ray outburst (5) in February 2008. Causality arguments imply that the emission region should have a spatial extent of less than $\approx 5 \delta R_{\mathrm{s}}$, where $\delta$ is the relativistic Doppler factor. This rules out explanations for the VHE $\gamma$-ray emission on the basis of (i) dark matter annihilation (15), (ii) cosmic-ray interactions with the matter in M87 (16), or (iii) the knots in the plasma jet (Fig. 1C). Leptonic $(17,18)$ and hadronic $(19)$ VHE $\gamma$-ray jet emission models have been proposed. However, the location of the emission region is still unknown. The nucleus $(20,21)$, the inner jet (22), or larger structures in the jet, such as the knot HST-1 (Fig. 1C), have been discussed as possible sites (14). Because the angular resolution of VHE experiments is of the order of $0.1^{\circ}$, the key to identifying the location of the VHE $\gamma$-ray emission lies in connecting it to measurements at other wave bands with considerably higher spatial resolutions. An angular resolution more than six orders of magnitude better (less than $6 \times 10^{-8}$ degrees, corresponding to approximately $30 R_{\mathrm{S}}$ in the case of M87) can be achieved with radio observations (Fig. 1).

We used the H.E.S.S. (23), MAGIC (24), and VERITAS (25) instruments to observe M87 during 50 nights between January and May 2008, accumulating over 95 hours of data (corrected for the detector dead times) in the energy range between $0.1 \mathrm{TeV}$ and several 10s of TeV. Simultaneously, we monitored M87 with the Very Long Baseline Array (26) (VLBA) at $43 \mathrm{GHz}$ with a resolution of $0.21 \times 0.43$ milli-arc sec $(27)$, corresponding to about $30 \times 60 R_{\mathrm{s}}$. During the first half of 2008, three x-ray pointings were performed with the Chandra satellite (28). Our light curves are shown in Fig. 2.

We detected multiple flares at VHE in February 2008 with denser sampling, following a trigger sent by MAGIC [ $\sim 23$ hours of the data published in (5)]. The short-term VHE variability, first observed in 2005 (2), is clearly confirmed and the flux reached the highest level observed 
so far from M87, amounting to more than $10 \%$ of that of the Crab Nebula. At x-ray frequencies the innermost knot in the jet (HST-1) is found in a low state, whereas in mid-February 2008, the nucleus was found in its highest $\mathrm{x}$-ray flux state since 2000 (28). This is in contrast to the 2005 VHE $\gamma$-ray flares (2), which happened after an increase of the x-ray flux of HST-1 over several years (29), allowing speculation that HST-1 might be the source of the VHE $\gamma$-ray emission (14); no $43 \mathrm{GHz}$ radio observations were obtained at that time. Given its low x-ray flux in 2008, HST-1 is an unlikely site of the 2008 VHE flaring activity.

Over at least the following two months, until the VLBA monitoring project ended, the 43$\mathrm{GHz}$ radio flux density from the region within 1.2 milli-arc sec of the core rose by $30 \%$, as compared with its level at the time of the start of the VHE flare, and by $57 \%$, as compared with the average level in 2007 (Fig. 2). The resolution of the 43-GHz images corresponds to $30 \times$ $60 R_{\mathrm{s}}$, and the initial radio flux density increase was located in the unresolved core. The region around the core brightened as the flare progressed (Fig. 3), suggesting that new components were emerging from the core. At the end of the observations, the brightened region extended about 0.77 milli-arc sec from the peak of the core, implying an average apparent velocity of $1.1 c$ (where $c$ is the speed of light), well under the approximately $2.3 c$ seen just beyond that distance in the first half of 2007. Astrometric results obtained as part of the VLBA monitoring program show that the position of the M87 radio peak, relative to M84, did not move by more than $\sim 6 R_{\mathrm{s}}$ during the flare, suggesting that the peak emission corresponds to the nucleus of M87.

Because VHE, x-ray, and radio flares of the observed magnitude are uncommon, the fact that they happen together (chance probability of $P<0.5 \%$, SOM text) is good evidence that they are connected. This is supported by our joint modeling of the VHE and radio light curves: The observed pattern can be explained by an event in the central region causing the VHE flare. The plasma travels down the jet, and the effect of synchrotron self-absorption causes a delay of the observed peak in radio emission because the region is not transparent at radio energies at the beginning of the injection (SOM text, section 3). The VLBI structure of the flare, along with the timing of the VHE activity, imply that the VHE emission occurred in a region that is small when compared with the VLBA resolution. Unless a source of infrared radiation is located very close to the central black hole, which is not supported by current observations (30), teraelectron volt $\gamma$-ray photons can escape the central region of M87 without being heavily absorbed through $\mathrm{e}^{+} \mathrm{e}^{-}$pair production $(20,21)$.

The light curve might indicate a rise in radio flux above the range of variations observed in the past, starting before the first VHE flare was detected. This could imply that the radio emis-

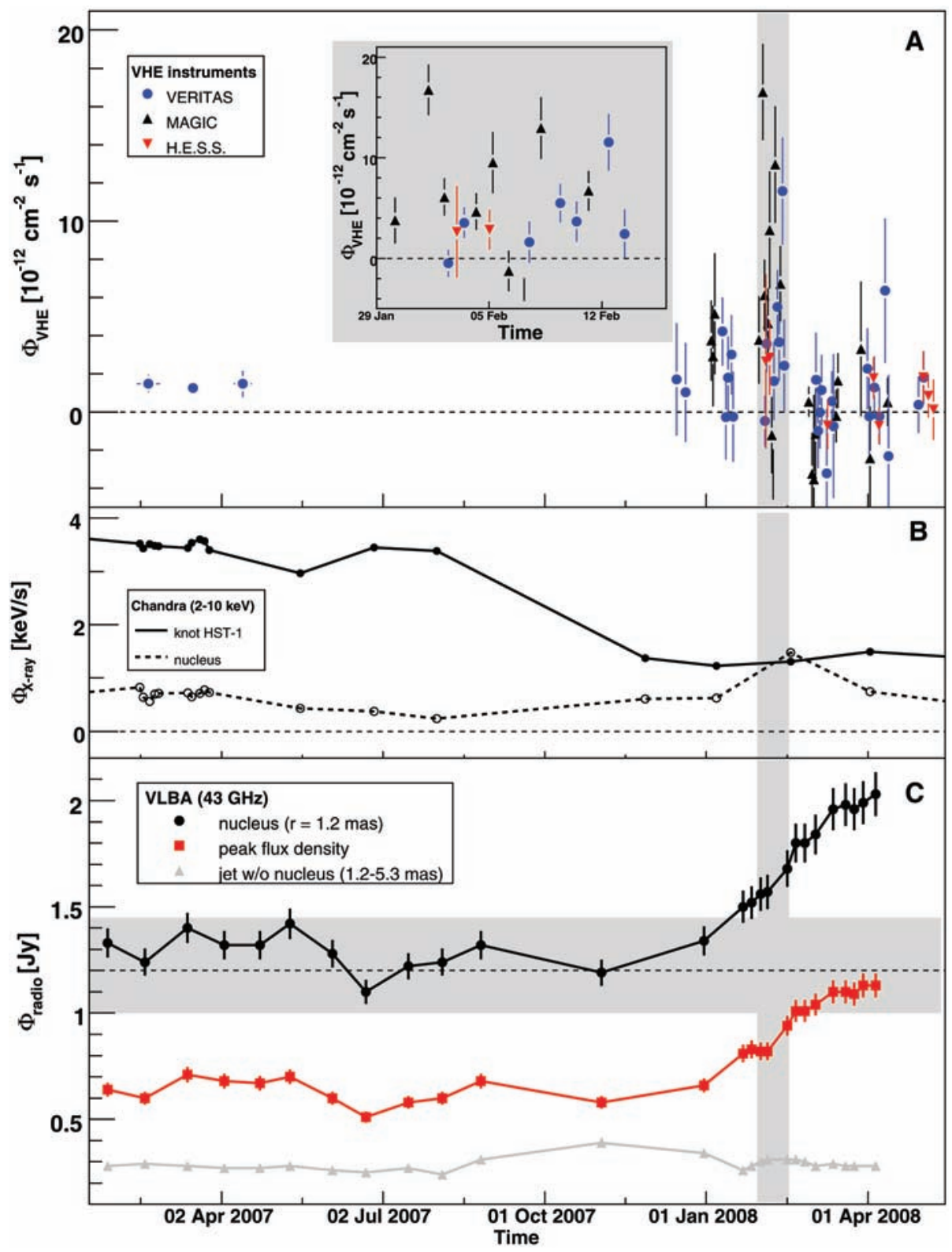

Fig. 2. Combined M87 light curves from 2007 to 2008. (A) VHE $\gamma$-ray fluxes $(E>0.35 \mathrm{TeV}$, nightly average), showing the H.E.S.S., MAGIC, and VERITAS data. The fluxes with statistical errors (1 SD) were calculated assuming a power-law spectral shape of $d N / d E \propto E^{-2.3}$. Monthly binned archival VERITAS data taken in 2007 are also shown (4). The systematic uncertainty in the flux calibration between the experiments was estimated to be on the order of $20 \%$, based on Crab Nebula data. The regular gaps in the light curve correspond to phases of full moon during which no observations were possible. The inlay shows a zoomed version of the flaring activity in February 2008; the time span is indicated by the gray vertical box in all panels. (B) Chandra x-ray measurements (2 to $10 \mathrm{keV}$ ) of the nucleus and the knot HST-1 (28). (C) Flux densities from the 43-GHz VLBA observations are shown for (i) the nucleus (circular region with radius $r=1.2$ milli-arc sec $=170 R_{\mathrm{s}}$ centered on the peak flux), (ii) the peak flux (VLBA resolution element), and (iii) the flux integrated along the jet between distances of $r=1.2$ to 5.3 milli-arc sec (compare with Fig. 3). The error bars correspond to $5 \%$ of the flux. The shaded horizontal area indicates the range of fluxes from the nucleus before the 2008 flare. Whereas the flux of the outer regions of the jet does not change substantially, most of the flux increase results from the region around the nucleus.

sion is coming from portions of the jet launched from further out in the accretion disk than that responsible for the VHE emission. However, it is difficult to derive a quantitive statement on this, because no VHE data were taken in the week previous to the flaring. Thus, an earlier start of the VHE activity cannot be excluded either.
A possible injection of plasma at the base of the jet observed at optical and x-ray energies, with a delayed passage through the radio core $\sim 10^{4} R_{\mathrm{s}}$ further down the jet - interpreted as a standing shock and accompanied by an increase in radio emission - has been discussed in the case of BL Lac (10) (with evidence for VHE emission, 

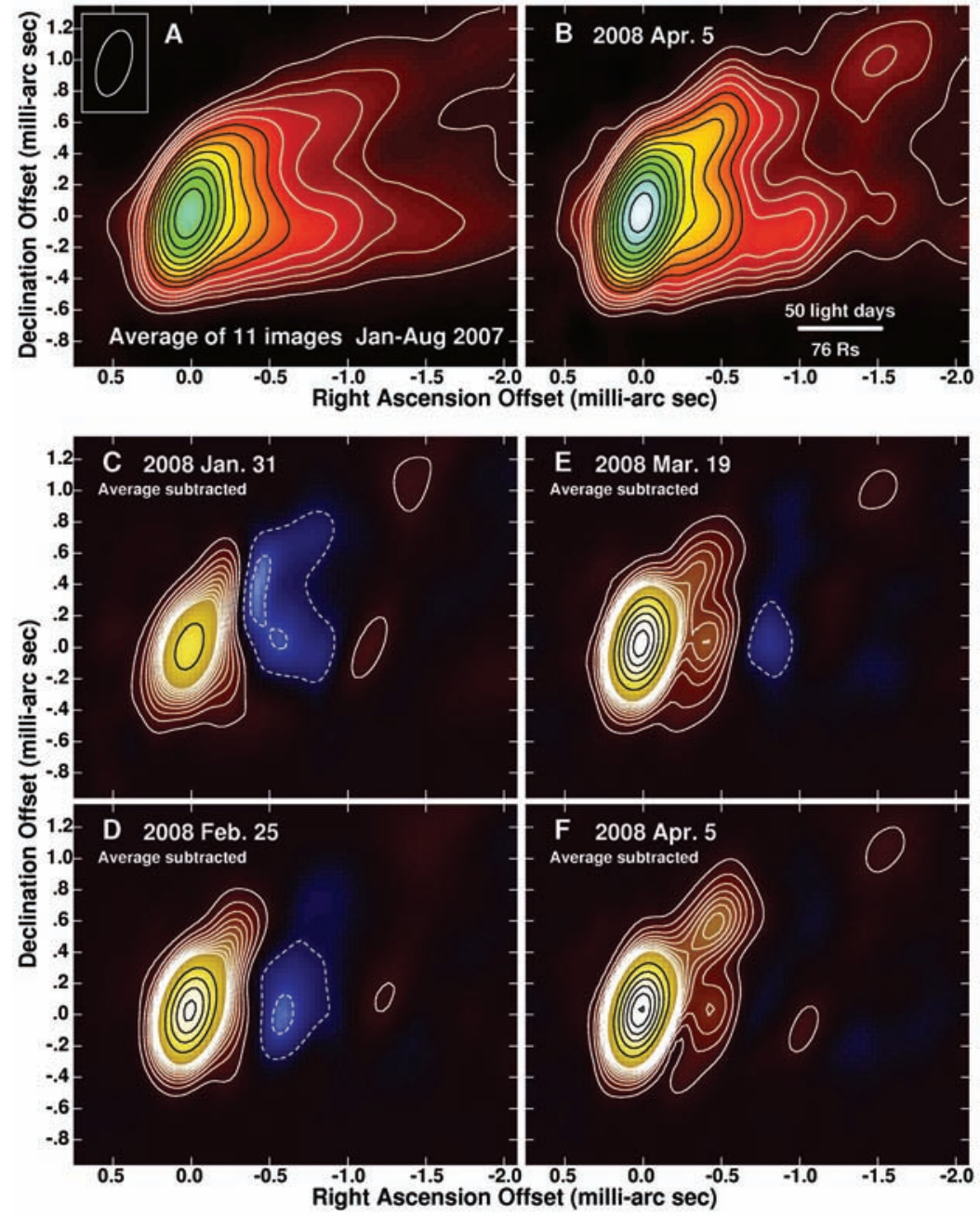

Fig. 3. VLBA images of $M 87$ at $43 \mathrm{GHz}$. (A) Average (hence smoothed) of 11 images from data taken between January and August 2007, well before the VHE and radio flare. The contour levels start at 5 , $10,14.3$, and $20 \mathrm{~m}$ ]y per beam and increase from there by factors of $\sqrt{2}$. The restoring beam used for all of the images is $0.21 \times 0.43$ milli-arc sec $\left(30 \times 60 R_{s}\right)$ elongated in position angle $-16^{\circ}$, as shown by the ellipse in the upper left corner. (B) Image from 5 April 2008, with the same contours and colors as in (A). The linear scale in light days and Schwarzschild radii is also shown. (C to $\mathbf{F}$ ) Difference images for observations during the period of the radio flare, showing its effects. These were made by subtracting the average image (A) from the individual epoch images. The contours are linear with 10 (white) at intervals of $7 \mathrm{~m}$ jy per beam, followed by the rest (black) at intervals of $70 \mathrm{~m}$ jy per beam; negative contours are indicated by dashed lines. At the time of the VHE flare, the core flux density was already above the average, but the region of the jet between -0.5 and -1.0 milli-arc sec RA offset was below average, suggesting that there had been a period of below-normal activity leading up to the flare and that the radio flare may have begun before the VHE flare. The sequence shows the substantial rise in the core flux density and the appearance of enhanced emission along the inner jet.

see SOM text for more details). M87 is much closer than BL Lac and has a much more massive black hole, allowing the VLBA to start resolving the jet collimation region whose size, from general relativistic magnetohydrodynamic simulations (31), is thought to extend over $\sim 1000 R_{\mathrm{s}}$. In the case of M87, the radio core does not appear to be offset by more than the VLBA resolution of $\sim 50 R_{\mathrm{S}}$ from the black hole (see SOM text), and the jet has a larger angle to the line of sight than in BL Lac. Thus, the coincidence of the VHE and radio flares (separated in photon frequency by 16 orders of magnitude), constrains the VHE emission to occur well within the jet collimation region.

\section{References and Notes}

1. J. A. Gaidos et al., Nature 383, 319 (1996).

2. F. Aharonian et al., Science 314, 1424 (2006); published online 26 October 2006 (10.1126/science.1134408).

3. F. Aharonian et al., Astron. Astrophys. 403, L1 (2003).

4. V. A. Acciari et al., Astrophys. J. 679, 397 (2008),

5. J. Albert et al., Astrophys. J. 685, L23 (2008).

6. K. Gebhardt, ]. Thomas, Astrophys. J., in press; preprint available at http://arxiv.org/abs/0906.1492 (2009)

7. The Schwarzschild radius of a black hole with the mass $m$ is defined as $R_{\mathrm{s}}=2 \mathrm{Gm} / \mathrm{c}^{2}$, where $G$ is the gravitational constant. The Schwarzschild radius defines the event horizon of the black hole

8. A. S. Wilson, Y. Yang, Astrophys. J. 568, 133 (2002).

9. W. Junor, J. A. Biretta, M. Livio, Nature 401, 891 (1999).

10. A. P. Marscher et al., Nature 452, 966 (2008).

11. C. Ly, R. C. Walker, W. Junor, Astrophys. J. 660, 200 (2007).

12. Y. Y. Kovalev, M. L. Lister, D. C. Homan, K. I. Kellermann, Astrophys. J. 668, L27 (2007).

13. ]. A. Biretta, W. B. Sparks, F. Macchetto, Astrophys. J. 520, 621 (1999)

14. C. C. Cheung, D. E. Harris, L. Stawarz, Astrophys. J. 663 L65 (2007).

15. E. A. Baltz et al., Phys. Rev. D 61, 023514 (2000).

16. C. Pfrommer, T. A. Enßlin, Astron. Astrophys. 407, L73 (2003)

17. M. Georganopoulos, E. S. Perlman, D. Kazanas, Astrophys. J. 634, L33 (2005).

18. J.-P. Lenain et al., Astron. Astrophys. 478, 111 (2008).

19. A. Reimer, R. J. Protheroe, A.-C. Donea, Astron. Astrophys. 419, 89 (2004).

20. A. Neronov, F. A. Aharonian, Astrophys. J. 671, 85 (2007).

21. F. M. Rieger, F. A. Aharonian, Int. J. Mod. Phys. D 17, 1569 (2008).

22. F. Tavecchio, G. Ghisellini, Mon. Not. R. Astron. Soc. 385 L98 (2008).

23. F. Aharonian et al., Astron. Astrophys. 457, 899 (2006).

24. ]. Albert et al., Astrophys. J. 674, 1037 (2008).

25. V. A. Acciari et al., Astrophys. J. 679, 1427 (2008).

26. P. J. Napier et al., Proc. IEEE 82, 658 (1994).

27. R. C. Walker, C. Ly, W. Junor, P. E. Hardee, ]. Phys. Conf. Ser. 131, 012053 (2008).

28. D. E. Harris, C. C. Cheung, L. Stawarz, ]. A. Biretta, E. S. Perlman, Astrophys. J. 699, 305 (2009).

29. D. E. Harris et al., Astrophys. J. 640, 211 (2006).

30. E. S. Perlman et al., Astrophys. J. 663, 808 (2007).

31. J. C. McKinney, Mon. Not. R. Astron. Soc. 368, 1561 (2006).

32. F. N. Owen, ]. A. Eilek, N. E. Kassim, Astrophys. J. 543, $611(2000)$

33. H.E.S.S.: We thank the Namibian authorities and the University of Namibia for support in facilitating the construction and operation of H.E.S.S. We also thank the German Ministry for Education and Research (BMBF), the Max Planck Society, the French Ministry for Research, CNRS-IN2P3 and the Astroparticle Interdisciplinary Programme of CNRS, the U.K. Science and Technology Facilities Council (STFC), the Institute of Particle and Nuclear Physics of the Charles University, the Polish Ministry of Science and Higher Education, and the South African Department of Science and Technology and National Research Foundation for support. We appreciate the excellent work of the technical support staff in Berlin, Durham, Hamburg, Heidelberg, Palaiseau, Paris, and Saclay and also in Namibia in the construction and operation of the equipment. MAGIC: We thank the Instituto de Astrofísica de Canarias for the excellent working conditions at the Observatorio del Roque de los Muchachos in La Palma, as well as the German BMBF and MPG, the Italian INFN and Spanish Ministerio de Ciencia e Innovación. This work was also supported by ETH research grant TH 34/043, the Polish MniSzW grant N N203 390834, and the Young Investigators Program of the Helmholtz Gemeinschaft. VERITAS: This research is supported by grants from the U.S. Department of Energy, NSF, the Smithsonian Institution, the Natural Sciences and Engineering Research Council of Canada, Science Foundation Ireland, and the STFC in the U.K. We acknowledge the excellent work of the technical support staff at the Fred Lawrence Whipple Observatory and the collaborating institutions in the construction and operation of the instrument. VLBA: The Very Long Baseline Array is operated by the National Radio Astronomy Observatory, a facility of NSF, operated under cooperative agreement by Associated Universities, Inc.

*The VERITAS Collaboration: V. A. Acciari, ${ }^{1}$ E. Aliu, ${ }^{2}$ T. Arlen, ${ }^{3}$ M. Bautista, ${ }^{4}$ M. Beilicke, ${ }^{5}+$ W. Benbow ${ }^{1}$ S. M. Bradbury, ${ }^{6}$ ]. H. Buckley, ${ }^{5}$ V. Bugaev, ${ }^{5}$ Y. Butt, ${ }^{7}$ K. Byrum, ${ }^{8}$ A. Cannon, ${ }^{9}$ O. Celik, ${ }^{3}$ A. Cesarini, ${ }^{10}$ Y. C. Chow, ${ }^{3}$ L. Ciupik, ${ }^{11}$ P. Cogan, ${ }^{4}$ W. Cui, ${ }^{12}$ R. Dickherber, ${ }^{5}$ S. J. Fegan, ${ }^{3}$ ]. P. Finley, ${ }^{12}$ P. Fortin, ${ }^{13}$ L. Fortson, ${ }^{11}$ A. 
Furniss, ${ }_{1}^{14}$ D. Gall, ${ }^{12}$ G. H. Gillanders, ${ }^{10}{ }^{1}$ J. Grube, ${ }^{9}$ R. Guenette, ${ }^{4} \mathrm{G}$ Gyuk, ${ }^{11}$ D. Hanna ${ }^{4}$ ]. Holder, ${ }^{2}$ D. Horan, ${ }^{15}$ C. M. Hui ${ }^{16}{ }^{16}$ T. B. Humensky, ${ }^{17}$ A. Imran, ${ }^{18}$ P. Kaaret, ${ }^{19}$ N. Karlsson, ${ }^{11}$ D. Kieda, ${ }^{16}$ ]. Kildea, ${ }^{1}$ A. Konopelko, ${ }^{20}$ H. Krawczynski, ${ }^{5} \dagger$ F. Krennrich, ${ }^{18}$ M. J. Lang, ${ }_{10}^{10}$ S. LeBohec, ${ }^{16}$ G. Maier, ${ }^{4}$ A. McCann, ${ }^{4}$ M. McCutcheon, ${ }^{4}$ ]. Millis, ${ }^{21}$ P. Moriarty, ${ }^{22}$ R. A. Ong, ${ }^{3}$ A. N. Otte, ${ }^{14}$ D. Pandel, ${ }^{19}$ ]. S. Perkins, ${ }^{1}$ D. Petry, ${ }^{23}$ M. Pohl, ${ }^{18}$ J. Quinn, ${ }^{9}$ K. Ragan ${ }^{4}$ L. C. Reyes, ${ }^{24}$ P. T. Reynolds, ${ }^{25}$ E. Roache, ${ }_{1}^{1}$ E. Roache, ${ }^{1}$ H. J. Rose, ${ }^{6}$ M. Schroedter, ${ }^{18}$ G. H. Sembroski, ${ }^{12}$ A. W. Smith, ${ }^{8}$ S. P. Swordy, ${ }_{1}^{17}$ M. Theiling, ${ }^{1}$ ]. A. Toner, ${ }^{10}$ A. Varlotta, ${ }^{12}$ S. Vincent, ${ }^{16}$ S. P. Wakely, ${ }_{17}^{17}$ ]. E. Ward, ${ }^{9}$ T. C. Weekes, ${ }^{1}$ A. Weinstein, ${ }^{3}$ D. A. Williams, ${ }^{14}$ S. Wissel, ${ }^{17}$ M. Wood ${ }^{3}$

The VLBA 43 GHz M87 Monitoring Team: R. C. Walker, ${ }^{26} \dagger$ F. Davies, ${ }^{26,27}$ P. E. Hardee, ${ }^{28}$ † W. Junor, ${ }^{29}$ C. Ly ${ }^{30}$

The H.E.S.S. Collaboration: F. Aharonian, ${ }^{31,43}$ A. G. Akhperjanian, ${ }^{32}$ G. Anton, ${ }^{46}$ U. Barres de Almeida, ${ }^{38} \neq$ A. R. Bazer-Bachi, ${ }^{33} Y$. Becherini, ${ }^{42}$ B. Behera, ${ }^{44}$ K. Bernlöhr, ${ }^{31,35}$ A. Bochow, ${ }^{31}$ C. Boisson, ${ }^{36}$ J. Bolmont ${ }^{49}$ V. Borrel, ${ }^{33}$ J. Brucker, ${ }^{46}$ F. Brun, ${ }^{49}$ P. Brun, ${ }^{37}$ R. Bühler, ${ }^{31}$ T. Bulik, ${ }^{54}$ I. Büsching, ${ }^{39}$ T. Boutelier, ${ }^{47}$ P. M. Chadwick, ${ }^{38}$ A. Charbonnier, ${ }^{49}$ R. C. G. Chaves, ${ }^{31}$ A. Cheesebrough, ${ }^{38}$ L.-M. Chounet, ${ }^{40}$ A. C. Clapson, ${ }^{31}$ G. Coignet, ${ }^{41}$ M. Dalton, ${ }^{35}$ M. K. Daniel, ${ }^{38}$ I. D. Davids, ${ }^{39,52}$ B. Degrange, ${ }^{40}$ C. Deil, ${ }^{31}$ H. J. Dickinson, ${ }^{38}$ A. Djannati-Ataï, ${ }^{42}$ W. Domainko, ${ }^{31}$ L. O'C. Drury, ${ }^{43}$ F. Dubois, ${ }^{41} \mathrm{G}$ Dubus $^{47}$ ]. Dyks, ${ }^{54}$ M. Dyrda ${ }^{58}$ K. Egberts, ${ }^{31}$ D. Emmanoulopoulos, ${ }^{4{ }^{4}}$ P. Espigat, ${ }^{42} \mathrm{C}$. Farnier ${ }^{45} \mathrm{~F}$. Feinstein ${ }^{45}$ A. Fiasson ${ }^{45}$ A. Förster ${ }^{31} \mathrm{G}$. Fontaine ${ }^{40}$ M. Füßling, ${ }^{35}$ S. Gabici, ${ }^{43}$ Y. A. Gallant, ${ }^{45}$ L. Gérard, ${ }^{42} \mathrm{D}$. Gerbig, ${ }^{51}$ B. Giebels, ${ }^{40}$ ]. F. Glicenstein, ${ }^{37}$ B. Glück, ${ }^{46}$ P. Goret, ${ }^{37}$ D. Göhring, ${ }^{46}$ D. Hauser ${ }^{44} \mathrm{M}$. Hauser ${ }_{,}^{44} \mathrm{~S}$. Heinz, ${ }^{46} \mathrm{G}$. Heinzelmann, ${ }^{34}$ G. Henni, ${ }^{47}$ G. Hermann, ${ }^{31}$ ]. A. Hinton, ${ }^{55}$ A. Hoffmann, ${ }^{48}$ W. Hofmann ${ }^{31}$ M. Holleran ${ }^{39}$ S. Hoppe, ${ }^{31}$ D. Horns ${ }^{34}$ A. Jacholkowska, ${ }^{49}$ O. C. de Jager, ${ }^{39}$ C. Jahn, ${ }^{46}$ I. Jung, ${ }^{46}$ K. Katarzyńnki, ${ }^{57}$ U. Katz ${ }^{46} \mathrm{~S}$ Kaufmann ${ }^{44}$ E. Kendziorra, ${ }^{48} \mathrm{M}$. Kerschhaggl ${ }^{35}{ }^{35}$. Khangulyan, ${ }^{3}$ B. Khélifi, ${ }^{40}$ D. Keogh ${ }^{38}$ W. Kluźniak, ${ }^{54}$ T. Kneiske, ${ }^{34}$ Nu. Komin ${ }^{37} \mathrm{~K}$. Kosack, ${ }^{31}$ G. Lamanna, ${ }^{41}$ J.P. Lenain, ${ }^{36}$ T. Lohse, ${ }^{35}$ V. Marandon, ${ }^{42}$ J. M. Martin ${ }^{36}$ O. Martineau-Huynh ${ }^{49}$ A. Marcowith ${ }^{45}$ D. Maurin ${ }^{49}$ T. J. L. McComb, ${ }^{38}$ M. C. Medina, ${ }^{36}$ R. Moderski, ${ }^{54}$ E. Moulin, ${ }^{37}$ M. Naumann-Godo, ${ }^{40}$ M. de Naurois, ${ }^{49}$ D. Nedbal, ${ }^{50}$ D. Nekrassov, ${ }^{31}$ B. Nicholas, ${ }^{56}$ ]. Niemiec ${ }_{,}^{58}$ S. ]. Nolan ${ }_{1}^{38}$ S. Ohm, ${ }^{31}$ ].-F. Olive, ${ }^{33}$ E. de Oña Wilhelmi, ${ }^{42,59}$ K. J. Orford, ${ }^{38}$ M. Ostrowski, ${ }^{53}$ M. Panter, ${ }^{31}$ M. Paz Arribas, ${ }^{35}$ G. Pedaletti, ${ }^{44}$ G. Pelletier, ${ }^{47}$ P.-O. Petrucci, ${ }^{47}$ S. Pita, ${ }^{42}$ G. Pühlhofer, ${ }^{44}$ M. Punch, ${ }^{42}$ A. Quirrenbach, ${ }^{44}$ B. C. Raubenheimer, ${ }^{39}$ M. Raue, ${ }^{31,59}$ † S. M. Rayner, ${ }^{38}$ M. Renaud, ${ }^{42,31}$ F. Rieger, ${ }^{31,59}{ }^{\prime}$ ]. Ripken ${ }^{34}$ L. Rob ${ }^{50}$ S. Rosier-Lees ${ }^{41}$ G. Rowell ${ }^{56}$ B. Rudak ${ }^{54}$ C. B. Rulten, ${ }^{38}$ ]. Ruppel, ${ }^{51}$ V. Sahakian, ${ }^{32}$ A. Santangelo, ${ }^{48}$ R. Schlickeiser, ${ }^{51}$ F. M. Schöck ${ }^{46}$ R. Schröder, ${ }^{51}$ U. Schwanke, ${ }^{35}$ S. Schwarzburg, ${ }^{48}$ S. Schwemmer, ${ }^{44}$ A. Shalchi, ${ }^{51}$ M. Sikora, ${ }^{54}$ J. L. Skilton, ${ }^{55}$ H. Sol, ${ }^{36}$ D. Spangler, ${ }^{38}$ Ł. Stawarz ${ }^{53}$ R. Steenkamp, ${ }^{52}$ C. Stegmann, ${ }^{46}$ F. Stinzing, ${ }^{46}$ G. Superina, ${ }^{40}$ A. Szostek, ${ }^{53,47}$ P. H. Tam, ${ }^{44}$ J.-P. Tavernet, ${ }^{49}$ R. Terrier, ${ }^{42}$ O. Tibolla, ${ }^{3,44}$ M. Tluczykont, ${ }^{34}$ C. van Eldik, ${ }^{31} G$. Vasileiadis, ${ }^{45}$ C. Venter, ${ }^{39}$ L. Venter, ${ }^{36}$ ]. P. Vialle, ${ }^{41}$ P. Vincent ${ }^{49} \mathrm{M}$. Vivier, ${ }^{37}$ H. J. Völk, ${ }^{31}$ F. Volpe, ${ }^{31,40,59}$ S. ]. Wagner, ${ }^{44}$ M. Ward ${ }^{38}$ A. A. Zdziarski, $^{54}$ A. Zech ${ }^{36}$

The MAGIC Collaboration: H. Anderhub, ${ }^{60}$ L. A. Antonelli, ${ }^{61} \mathrm{P}$. Antoranz, ${ }^{62}$ M. Backes, ${ }^{63}$ C. Baixeras, ${ }^{64}$ S. Balestra, ${ }^{62}$ ]. A. Barrio, ${ }^{62}$ D. Bastieri, ${ }^{65}$ ]. Becerra González, ${ }^{66}$ ]. K. Becker ${ }^{63}$ W. Bednarek, ${ }^{67}$ K. Berger ${ }^{67}$ E. Bernardini, ${ }^{68}$ A. Biland, ${ }^{60}$ R. K. Bock, ${ }^{65,69}{ }^{6}$. Bonnoli, ${ }^{70}$ P. Bordas, ${ }^{71}$ D. Borla Tridon, ${ }^{69}$ V. Bosch-Ramon, ${ }^{71}$ D. Bose, ${ }^{62}$ ।. Braun, ${ }^{60}$ T. Bretz, ${ }^{72}$ I. Britvitch, ${ }^{60}$ M. Camara, ${ }^{62}$ E. Carmona ${ }^{69} \mathrm{~S}$. Commichau, ${ }^{60}$ ]. L. Contreras, ${ }^{62}$ ]. Cortina, ${ }^{73}$ M. T. Costado, ${ }^{66,74}$ S. Covino, ${ }^{61}$ V. Curtef, ${ }^{63}$ F. Dazzi, ${ }^{75} \S$ A. De Angelis, ${ }^{75}$ E. De Cea del Pozo, ${ }^{76} \mathrm{C}$. Delgado Mendez, ${ }^{66}$ R. De los Reyes, ${ }^{62}$ B. De Lotto, ${ }^{75} \mathrm{M}$. De Maria, ${ }^{75}$ F. De Sabata, ${ }^{75}$ A. Dominguez, ${ }^{77}$ D. Dorner, ${ }^{60}$ M. Doro, ${ }^{65}$ D. Elsaesser, ${ }^{72} \mathrm{M}$. Errando, ${ }^{73}$ D. Ferenc, ${ }^{78}$ E. Fernández, ${ }^{73}$ R. Firpo, M. V. Fonseca, ${ }^{62}$ L. Font, ${ }^{64} \mathrm{~N}$. Galante, ${ }^{69}$ R. J. García López, ${ }^{66,74}$ M. Garczarczyk, ${ }^{73}$ M. Gaug ${ }^{66}$ F. Goebel, ${ }^{69} \|$ D. Hadasch, ${ }^{64}$ M. Hayashida, ${ }^{69}$ A. Herrero, ${ }^{6,74}$ D. Hildebrand, ${ }^{60}$ D. Höhne-Mönch, ${ }^{72}$ J. Hose, ${ }^{69}$ C. C. Hsu, ${ }^{69}$ T. Jogler, ${ }^{69}$ D. Kranich, ${ }^{60}$ A. La Barbera, ${ }^{61}$ A. Laille, $^{78}$ E. Leonardo, ${ }^{70}$ E. Lindfors, ${ }^{79}$ S. Lombardi, ${ }^{65}$ F. Longo, ${ }^{75} \mathrm{M}$. López, ${ }^{65}$ E. Lorenz, ${ }^{60,69}$ P. Majumdar, ${ }^{68}$ G. Maneva, ${ }^{80} \mathrm{~N}$. Mankuzhiyil, K. Mannheim ${ }^{72}$ L. Maraschi, ${ }^{61}$ M. Mariotti, ${ }^{55}$ M. Martínez, ${ }^{73}$ D. Mazin, ${ }^{73}+$ M. Meucci, ${ }^{70}$ ]. M. Miranda, ${ }^{62}$ R. Mirzoyan, ${ }^{69}$ H. Miyamoto, ${ }^{69}$ J. Moldón, ${ }^{71}$ M. Moles, ${ }^{77}$ A. Moralejo, ${ }^{73}$ D. Nieto, ${ }^{62}$ K. Nilsson, ${ }^{79}$ ]. Ninkovic, ${ }^{69}$ I. Oya, ${ }^{62}$ R. Paoletti, ${ }^{70}$ J. M. Paredes, ${ }^{71}$ M. Pasanen, ${ }^{79}$ D. Pascoli, ${ }^{65}$ F. Pauss, ${ }^{60}$ R. G. Pegna, ${ }^{70}$ M. A. Perez-Torres, ${ }^{77}$ M. Persic, $^{75,81}$ L. Peruzzo, ${ }^{65}$ F. Prada, ${ }^{77}$ E. Prandini, ${ }^{65}$ N. Puchades, ${ }^{73}$ I. Reichardt, ${ }^{73}$ W. Rhode ${ }^{63}$ M. Ribó, ${ }^{71}$ J. Rico, ${ }^{73,82}$ M. Rissi, ${ }^{60}$ A Robert, ${ }^{64}$ S. Rügamer, ${ }^{72}$ A. Saggion, ${ }^{65}$ T. Y. Saito, ${ }^{69}$ M. Salvati, ${ }^{61}$ M. Sanchez-Conde, ${ }^{77}$ K. Satalecka, ${ }^{68} \mathrm{~V}$. Scalzotto ${ }^{65} \mathrm{~V}$. Scapin ${ }^{75} \mathrm{~T}$. Schweizer, ${ }^{69}$ M. Shayduk, ${ }^{69}$ S. N. Shore, ${ }^{83}$ N. Sidro, ${ }^{73}$ A. Sierpowska-Bartosik, ${ }^{76}$ A. Sillanpää, ${ }^{79}$ ]. Sitarek, ${ }^{67,69}$ D. Sobczynska, ${ }^{67}$
F. Spanier, ${ }^{72}$ A. Stamerra, ${ }^{70}$ L. S. Stark, ${ }^{60}$ L. Takalo, ${ }^{79}$ F. Tavecchio, ${ }^{61}$ P. Temnikov, ${ }^{80}$ D. Tescaro, ${ }^{73}$ M. Teshima, ${ }^{69}$ D. F. Torres, ${ }^{76,82}$ N. Turini, $^{70}$ H. Vankov, ${ }^{80}$ R. M. Wagner, ${ }^{69}+$ V. Zabalza, ${ }^{71}$ F. Zandanel, ${ }^{77}$ R. Zanin, ${ }^{73}$ ]. Zapatero ${ }^{64}$

${ }^{1}$ Fred Lawrence Whipple Observatory, Harvard-Smithsonian Center for Astrophysics, Amado, AZ 85645, USA. ${ }^{2}$ Department of Physics and Astronomy and the Bartol Research Institute, University of Delaware, Newark, DE 19716, USA. ${ }^{3}$ Department of Physics and Astronomy, University of California, Los Angeles, CA 90095, USA. ${ }^{4}$ Physics Department, McGill University, Montreal, Quebec H3A 2T8, Canada. ${ }^{5}$ Department of Physics, Washington University, St. Louis, MO 63130, USA. ${ }^{6}$ School of Physics and Astronomy, University of Leeds, Leeds, LS2 9]T, UK. ${ }^{7}$ Harvard-Smithsonian Center for Astrophysics, 60 Garden Street, Cambridge, MA 02138, USA. ${ }^{8}$ Argonne National Laboratory, 9700 South Cass Avenue, Argonne, IL 60439, USA. 'School of Physics, University College Dublin, Belfield, Dublin 4, Ireland. ${ }^{10}$ School of Physics, National University of Ireland, Galway, Ireland. ${ }^{11}$ Astronomy Department, Adler Planetarium and Astronomy Museum, Chicago, IL 60605, USA. ${ }^{12}$ Department of Physics, Purdue University, West Lafayette, IN 47907, USA. ${ }^{13}$ Department of Physics and Astronomy, Barnard College, Columbia University, NY 10027, USA. ${ }^{14}$ Santa Cruz Institute for Particle Physics and Department of Physics, University of California, Santa Cruz, CA 95064, USA. ${ }^{15}$ Laboratoire Leprince-Ringuet, Ecole Polytechnique, CNRS/IN2P3, F-91128 Palaiseau, France. ${ }^{16}$ Department of Physics and Astronomy, University of Utah, Salt Lake City, UT 84112, USA. ${ }^{17}$ Enrico Fermi Institute, University of Chicago, Chicago, IL 60637, USA. ${ }^{18}$ Department of Physics and Astronomy, lowa State University, Ames, IA 50011, USA. ${ }^{19}$ Department of Physics and Astronomy, University of lowa, Van Allen Hall, lowa City, IA 52242. USA. ${ }^{20}$ Department of Physics, Pittsburg State University, 1701 South Broadway, Pittsburg, KS 66762, USA. ${ }^{21}$ Department of Physics, Anderson University, 1100 East 5th Street, Anderson, IN 46012. ${ }^{22}$ Department of Life and Physical Sciences, Galway-Mayo Institute of Technology, Dublin Road, Galway, Ireland. ${ }^{23}$ European Southern Observatory, KarlSchwarzschild-Strasse 2, 85748 Garching, Germany. ${ }^{24}$ Kavli Institute for Cosmological Physics, University of Chicago, Chicago, IL 60637, USA. ${ }^{25}$ Department of Applied Physics and Instrumentation, Cork Institute of Technology, Bishopstown, Cork, Ireland. ${ }^{26}$ National Radio Astronomy Observatory (NRAO), Socorro, NM 87801, USA. ${ }^{27}$ Physics Department, 333 Workman Center, New Mexico Institute of Mining and Technology, 801 Leroy Place, Socorro, NM 87801, USA. ${ }^{28}$ Department of Physics and Astronomy, University of Alabama, Tuscaloosa, AL 35487, USA. ${ }^{29}$ ISR-2, MS-D436, Los Alamos National Laboratory, Los Alamos, NM 87545, USA. ${ }^{30}$ Department of Astronomy, University of California, Los Angeles, CA 90095-1547, USA. ${ }^{31}$ Max-Planck-Institut für Kernphysik, Post Office Box 103980, D-69029 Heidelberg, Germany. ${ }^{32}$ Yerevan Physics Institute, 2 Alikhanian Brothers Street, 375036 Yerevan, Armenia. ${ }^{33}$ Centre d'Etude Spatiale des Rayonnements, CNRS/UPS, 9 Avenue du Colonel Roche, BP 4346, F-31029 Toulouse Cedex 4, France. ${ }^{34}$ Universität Hamburg, Institut für Experimentalphysik, Luruper Chaussee 149, D-22761 Hamburg, Germany. ${ }^{35}$ Institut für Physik, HumboldtUniversität zu Berlin, Newtonstrasse. 15, D-12489 Berlin, Germany. ${ }^{36}$ Laboratoire Univers et Théories, Observatoire de Paris, CNRS, Université Paris Diderot, 5 Place Jules Janssen, 92190 Meudon, France. ${ }^{37}$ Institut de Recherche sur les lois Fondamentales de I'Univers/La Direction des Sciences de la Matière/Commissariat a l'Energie Atomique CE Saclay, F-91191 Gif-sur-Yvette, Cedex, France. ${ }^{38}$ University of Durham, Department of Physics, South Road, Durham DH1 3LE, UK. ${ }^{39}$ Unit for Space Physics, North-West University, Potchefstroom 2520, South Africa. ${ }^{40}$ Laboratoire Leprince-Ringuet, Ecole Polytechnique, CNRS/IN2P3, F-91128 Palaiseau, France. ${ }^{41}$ Laboratoire d'Annecy-le-Vieux de Physique des Particules, CNRS/IN2P3, 9 Chemin de Bellevue, BP 110 F-74941 Annecy-le-Vieux Cedex, France. ${ }^{42}$ UMR 7164 (CNRS, Université Paris VII, CEA, Observatoire de Paris); Astroparticule et Cosmologie, CNRS; Universite Paris 7 Denis Diderot, 10, rue Alice Domon et Leonie Duquet, F-75205 Paris Cedex 13, France. ${ }^{43}$ Dublin Institute for Advanced Studies, 5 Merrion Square, Dublin 2, Ireland. ${ }^{44}$ Landessternwarte, Universität Heidelberg, Königstuhl, D-69117
Heidelberg, Germany. ${ }^{45}$ Laboratoire de Physique Théorique et Astroparticules, Université Montpellier 2, CNRS/IN2P3, CC 70, Place Eugène Bataillon, F-34095 Montpellier Cedex 5, France. ${ }^{46}$ Universität Erlangen-Nürnberg, Physikalisches Institut, ErwinRommel-Strasse 1, D-91058 Erlangen, Germany. ${ }^{47}$ Laboratoire d'Astrophysique de Grenoble, Institut National des Sciences de l'Univers/CNRS, Université Joseph Fourier, BP 53, F-38041 Grenoble Cedex 9, France. ${ }^{48}$ Institut für Astronomie und Astrophysik, Universität Tübingen, Sand 1, D-72076 Tübingen, Germany. ${ }^{49}$ Laboratoire de Physique Nucléaire et des Hautes, Université Pierre et Marie Curie Paris 6, Université Denis Diderot Paris 7, CNRS/IN2P3, 4 Place Jussieu, F-75252, Paris Cedex 5, France. ${ }^{50}$ Charles University, Faculty of Mathematics and Physics, Institute of Particle and Nuclear Physics, V Holesovickách 2, 180 00, Prague, Czech Republic. ${ }^{51}$ Institut für Theoretische Physik, Lehrstuhl IV: Weltraum und Astrophysik, Ruhr-Universität Bochum, D-44780 Bochum, Germany. ${ }^{52}$ University of Namibia, Private Bag 13301, Windhoek, Namibia. ${ }^{53}$ Obserwatorium Astronomiczne, Uniwersytet Jagielloński, ulica Orla 171, 30-244 Kraków, Poland. ${ }^{54}$ Nicolaus Copernicus Astronomical Center, ulica Bartycka 18, 00-716 Warsaw, Poland. ${ }^{55}$ School of Physics and Astronomy, University of Leeds, Leeds LS2 9JT, UK. ${ }^{56}$ School of Chemistry and Physics, University of Adelaide, Adelaide 5005, Australia. ${ }^{57}$ Toruń Centre for Astronomy, Nicolaus Copernicus University, ulica Gagarina 11, 87100 Toruń, Poland. ${ }^{58}$ Instytut Fizyki Jadrowej PAN, ulica Radzikowskiego 152, 31-342 Kraków, Poland. ${ }^{59}$ European Associated Laboratory for Gamma-Ray Astronomy, jointly supported by CNRS and Max-Planck-Gessellschaft. ${ }^{60}$ ETH Zurich, $\mathrm{CH}-8093$ Switzerland. ${ }^{61}$ Istituto Nazionale di Astrofisica (INAF) National Institute for Astrophysics, I-00136 Rome, Italy. ${ }^{62}$ Universidad Complutense, E-28040 Madrid, Spain. ${ }^{63}$ Technische Universität Dortmund, D-44221 Dortmund, Germany. ${ }^{64}$ Universitat Autònoma de Barcelona, E-08193 Bellaterra, Spain. ${ }^{65}$ Università di Padova and Istituto Nazionale di Fisica Nucleare (INFN), I-35131 Padova, Italy. ${ }^{66}$ Instituto de Astrofísica de Canarias, E-38200 La Laguna, Tenerife, Spain. ${ }^{67}$ University of Łódź, PL-90236 Lodz, Poland. ${ }^{68}$ Deutsches Elektronen-Synchrotron, D-15738 Zeuthen, Germany. ${ }^{69} \mathrm{Max}$ Planck-Institut für Physik, D-80805 München, Germany. ${ }^{70}$ Università di Siena, and INFN Pisa, I-53100 Siena, Italy. ${ }^{71}$ Universitat de Barcelona [Institut de Ciencias del Cosmos/ Institut d'Estudis Espacials de Catalunya (IEEC)], E-08028 Barcelona, Spain. ${ }^{72}$ Universität Würzburg, D-97074 Würzburg, Germany. ${ }^{73}$ Institut de Física d'Altes Energies, Edifici Cn, Campus Universitat Autònoma de Barcelona, E-08193 Bellaterra, Spain. ${ }^{74}$ Departamento de Astrofísica, Universidad, E-38206 La Laguna, Tenerife, Spain. ${ }^{75}$ Università di Udine, and INFN Trieste, I-33100 Udine, Italy. ${ }^{76}$ Institut de Cienciès de l'Espai [IEEC-Consejo Superior de Investigaciones Científicas (CSIC)], E-08193 Bellaterra, Spain. ${ }^{77}$ Institut de Astrofísica de Andalucia (CSIC), E-18080 Granada, Spain. ${ }^{78}$ University of California, Davis, CA 95616-8677, USA. ${ }^{79}$ Tuorla Observatory, Turku University, Fl-21500 Piikkiö, Finland. ${ }^{80}$ Institute for Nuclear Research and Nuclear Energy, BG-1784 Sofia, Bulgaria. ${ }^{81}$ INAF/ Osservatorio Astronomico and INFN, 1-34143 Trieste, Italy. ${ }^{82}$ Institució Catalana de Recerca i Estudis Avançats, E-08010 Barcelona, Spain. ${ }^{83}$ Università di Pisa, and INFN Pisa, I-56126 Pisa, Italy.

†To whom correspondence should be addressed. E-mail: beilicke@physics.wustl.edu (M.B.); krawcz@wuphys.wustl.edu (H.K.); cwalker@aoc.nrao.edu (R.C.W.); phardee@bama.ua. edu (P.E.H.); martin.raue@mpi-hd.mpg.de (M.R.); mazin@ ifae.es (D.M.); robert.wagner@mpp.mpg.de (R.M.W.) †Supported by Coordenacao de Aperfeicoamento de Pessoal de Nivel Superior Foundation, Ministry of Education of Brazil. §Supported by INFN Padova.

IIDeceased.

\section{Supporting Online Material}

www.sciencemag.org/cgi/content/full/1175406/DC1 SOM Text

Figs. S1 and S2

References

24 April 2009; accepted 24 June 2009

Published online 2 July 2009;

10.1126/science. 1175406

Include this information when citing this paper. 